\title{
Latest Generation of Silicon Drift Detectors and Readout Electronics
}

Andreas Pahlke ${ }^{1}$, Florian Dams ${ }^{1}$, Reinhard Fojt ${ }^{1}$, Michael Fraczek ${ }^{1}$, Jürgen Knobloch ${ }^{1}$, Christian Luckey $^{1}$, Natsuki Miyakawa ${ }^{1}$ and Niklas Willems ${ }^{1}$

1. KETEK GmbH, Munich, Germany.

KETEK is the recognized market leader for Silicon Drift Detectors and offers a large variety of sensors with collimated areas starting at $7 \mathrm{~mm}^{2}$ up to $150 \mathrm{~mm}^{2}$ (active area 12 to $170 \mathrm{~mm}^{2}$ ). All detectors are available with different X-ray windows (Graphene/Beryllium/Polymer windows) and KETEK also offers multiple configurations of complete electronics to operate the detectors.

KETEK has further optimized the detector modules with regards to operation in high ambient temperatures and long-time stability. As a result, KETEK SDDs can be operated at ambient temperatures up to $+70^{\circ} \mathrm{C}$ still achieving chip temperatures down to $-50{ }^{\circ} \mathrm{C}$ leading to optimum detector performance for all detector sizes. The Peltier power is in parallel reduced by more than $50 \%$ compared to the previous thermoelectric cooler at a given fixed temperature. Long term stability tests revealed that the vacuum integrity of the new SDD modules is given even at permanent storage temperatures of $+80^{\circ} \mathrm{C}$.

The new cooling technology is available in combination with our vacuum encapsulated Graphene window detectors. This window consists of a $900 \mathrm{~nm}$ graphenic carbon without any support grid. Over the complete energy range the window achieves a better transmission than an $8 \mu \mathrm{m}$ Beryllium window. The window itself is light tight and there is no necessity to use any additional light blocking layers such as aluminium. The burst pressure of the new windows has been tested up to 5 bar and a burst pressure of 2 bar is guaranteed. To prove the reliability of the new windows, pressure cycle tests have been performed and first prototypes passed more than 10 million cycles (vacuum to one atmosphere) without any degradation of the window performance.

For low energy applications, detecting X-ray lines below $1 \mathrm{keV}$, KETEK offers the new technology in combination with a polymer AP3.3 windows and a Xenon gas backfill to minimize the heat transportation by gas convection. This configuration is available for the VITUS H7, H20, H30 and H50 with the cold side temperature being as low as $-60{ }^{\circ} \mathrm{C}$ at $+20^{\circ} \mathrm{C}$ heatsink temperature.

We will also present latest results of a $150 \mathrm{~nm}$ graphenic carbon layer window supported by a silicon grid (see figure 1). Again, no additional layers are needed to make the window light tight. The transmission is superior compared to a polymer window over the whole energy range.

Silicon Drift Detectors manufactured by KETEK reveal excellent low energy performance. Even detection of Lithium (at $53 \mathrm{eV}$ ) with windowless modules has been demonstrated (see figure 2). The energy resolution of the Carbon peak $(277 \mathrm{eV})$ is as good as $42 \mathrm{eV}$.

KETEK also offers all electronics necessary to operate the SDD modules including a state-of-the-art digital pulse processor. We will present the latest generation of KETEK electronics. 
References:

[1] S Huebner et al., IEEE Trans. Nucl. Sci. 62 (2) (2015), p. 588.

[2] S Huebner et al., Phys. Status Solidi B 252 (11) (2015), p. 2564.

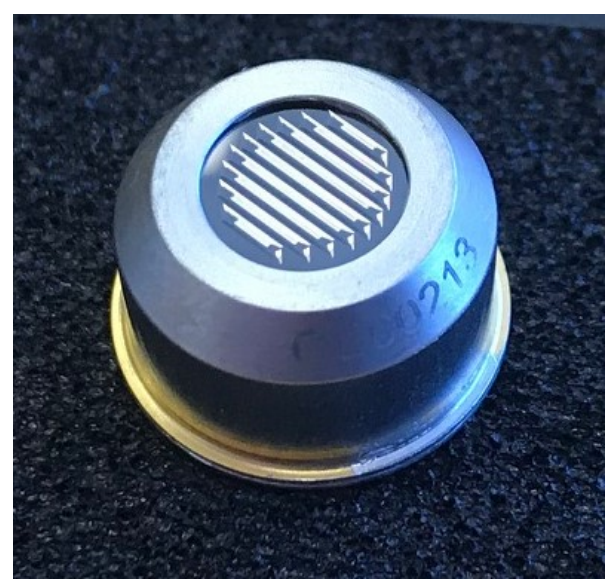

Figure 1. Vacuum encapsulated module with $150 \mathrm{~nm}$ graphenic window on a Silicon support grid

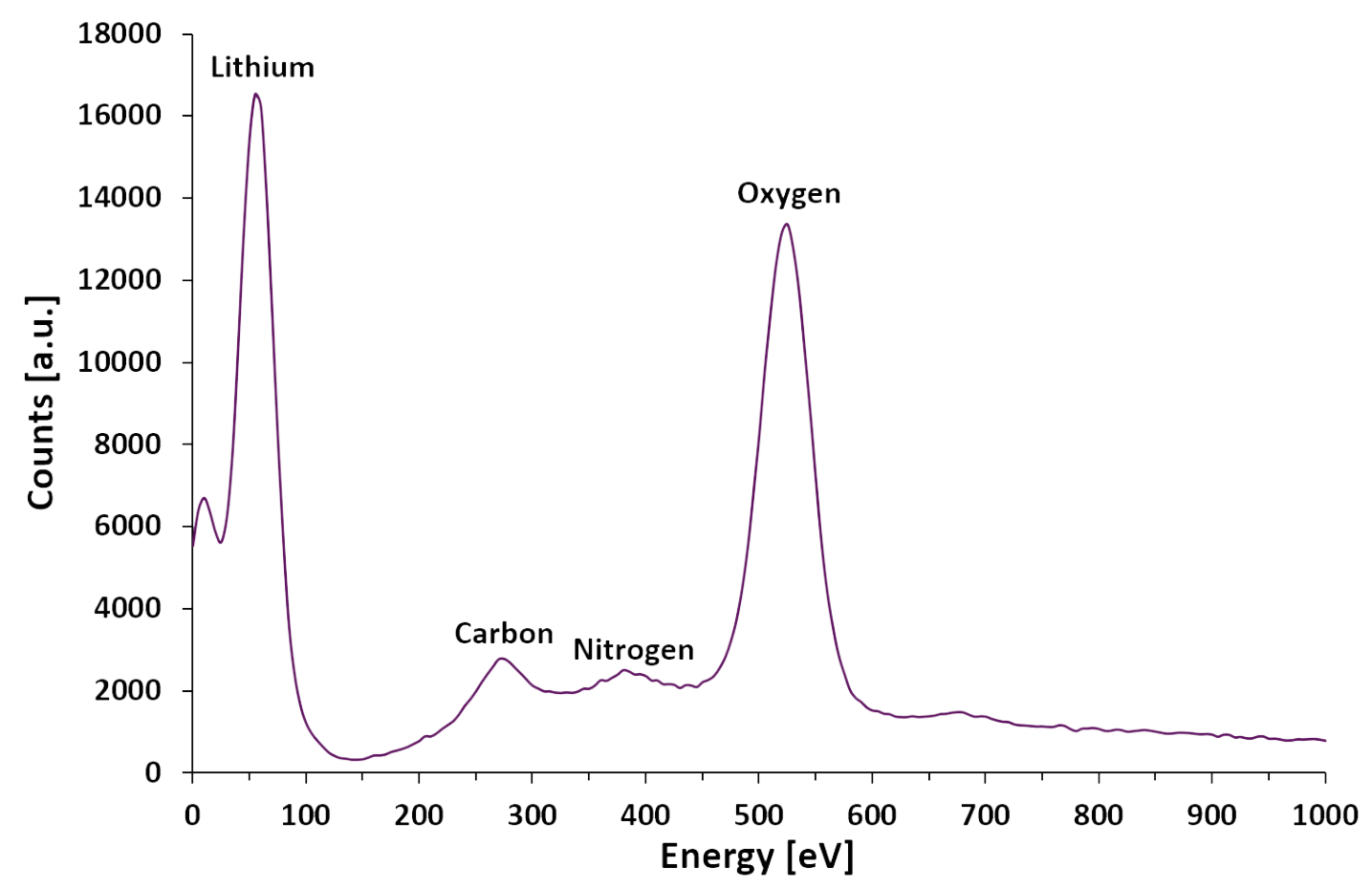

Figure 2. Low energy spectrum of KETEK SDDs showing the Lithium-K $\alpha$ peak 